\title{
EFFECT OF LUBRICATION ON THE PERFORMANCE AND DYNAMIC LOADS OF SCROLL COMPRESSORS
}

\author{
Jen-Jie Hsieh * \\ Department of Mechanical Engineering \\ National Taiwan University \\ Taipei, Taiwan 10617, R.O.C.
}

\author{
Wen-Shing Lee ** \\ Dept. of Air-Conditioning and Refrigeration Engineering \\ National Taipei University of Technology \\ Taipei, Taiwan 10626, R.O.C.
}

\author{
Wen-Fang Wu ${ }^{* * *} \quad$ Sih-Li Chen ${ }^{* * *}$ \\ Department of Mechanical Engineering \\ National Taiwan University \\ Taipei, Taiwan 10617, R.O.C.
}

\begin{abstract}
To study the effect of lubrication on the performance and dynamic loads of scroll compressors, a theoretical model is proposed in this paper. The model includes both thermal and dynamic analyses in which the lubrication theory is emphasized in particular. A computer simulation program is written accordingly, and the effects of different design parameters including oil flow rate, injection position, injection temperature and rotating speed are investigated. The output variables such as gas force, contact force, contact moment and thermal performance of the compressor are obtained. Some of the results are compared with experimentally measured data, and good agreements are found between the simulation results and the measured data. The applicability of the simulation program is thus verified. Analytical results from running this program can provide us valuable information on the design improvement of a scroll compressor.
\end{abstract}

Keywords : Scroll compressor, Lubrication system, Thermal analysis, Dynamic load.

\section{INTRODUCTION}

The concept of scroll compressor was originally proposed by Creux in 1905. It is a positive displacement compressor that utilities the continual variation of the space formed between the orbiting scroll and the fixed scroll to compress the gas. However, the production of scroll compressors on large scale did not start until the eighties when the manufacturing and related technology had been developed in succession. The scroll compressor has now been recognized as one of the best refrigerant compressors because of its many advantages [1].

Both the thermal process and lubrication mechanism affect the dynamic behavior of a scroll compressor as well as its extended noise and vibration. To study the heat transfer phenomena of a refrigerant scroll compressor, $\mathrm{Gu}$ and $\mathrm{Wu}$ [1] have carried out an experiment. The range of heat transfer data they obtained, however, is too narrow as compared with the theoretical result. Meyer and Thompson [2] have focused on the suction process and investigated experimentally the thermal performance of a scroll compressor. Suefjui, et al. [3] have studied the performance of a scroll compressor in detail by considering factors such as dynamic loss, leakage and heat transfer. The heat transfer model between the gas and scroll in the above studies, however, has been oversimplified.

In addition to the thermal/fluid process analysis, the dynamic analysis is also an important issue in designing a scroll compressor. The dynamic load caused by the combination of gas and contact force is affected greatly by the lubrication system of a scroll compressor. Short, and Rajewski [4] have collected statistical data for the lubrication system of refrigerant compressors and drawn several important conclusions about their influence on the dynamic load. The dynamic load analysis of a scroll compressor, however, has not been mentioned in particular in their paper.

In the present study, a general theoretical model accompanying with its computer simulation program is

\footnotetext{
* Graduate Student ** Assistant Professor ** Professor
} 
developed to evaluate the effect of lubrication on the performance and dynamic loads of a scroll compressor. The comparison results between present model and result will be compared with experimentally measured data justify the accuracy of the model. Once the model is justified successfully, the implementation of design for a certain type of scroll compressor can be achieved by simply carrying out the theoretical simulation program and making the appropriate decision on the numerical results.

\section{THERMAL ANAL YSIS}

The compression room of a scroll compressor lies in the space sealed by the fixed scroll and the orbiting scroll. When the compressor is running, several compression chambers exist simultaneously, and the refrigerant flows into neighboring chambers in a sequential order. To simulate the gas inside the compression room and reflect the real compression process, many quantities and/or factors such as charge gas, discharge gas, leakage, oil seal as well as heat and mass transfer are all considered in the present analysis. Nevertheless, owing to its complexity, a few assumptions are still needed. First, it is assumed that refrigerant is distributed uniformly in a compression chamber. Under this circumstance, the pressure, temperature and density of the refrigerant are all constants inside the chamber. The temperature of lubrication oil in the compression chamber is also constant. Secondly, the Iubrication oil is assumed incompressible and its density depends only on the temperature inside the compression chamber. Thirdly, the oil exchanges heat with the refrigerant but does not alter its phase.

There are two kinds of fluid, gas and lubricant exist simultaneously in the compression room. Their states and properties are not the same and need to be described separately. From conservation of mass and energy, the governing equations for the refrigerant can be written as

$$
\frac{d m_{g}}{d \theta_{m}}=\frac{1}{\omega_{m}}\left(\sum_{k=1}^{n 1} \dot{m}_{g i k}-\sum_{k=1}^{n 2} \dot{m}_{g o k}\right)
$$

and

$$
\begin{aligned}
\frac{d T_{g}}{d \theta_{m}} & =\frac{1}{\omega_{m} m_{g} C_{v, g}}\left\{-T_{g}\left(\frac{\partial P}{\partial T}\right)_{v}\left[\omega_{m} \frac{d V}{d \theta_{m}}+\frac{1}{\rho_{l}}\left(\dot{m}_{l o}-\dot{m}_{l i}\right)\right]\right. \\
& +\sum_{k=1}^{n 1} i_{g i k} \dot{m}_{g i k}+\left[\frac{V-m_{l} / \rho_{l}}{m_{g}} T_{g}\left(\frac{\partial P}{\partial T}\right)_{v}-i_{g}\right] \sum_{k=1}^{n_{1}} \dot{m}_{g i k} \\
& \left.-\frac{V-m_{l} / \rho_{l}}{m_{g}} T_{g}\left\{\frac{\partial P}{\partial T}\right)_{v} \sum_{k=1}^{n 2} \dot{m}_{g o k}-h A\left(T_{g}-T_{l}\right)\right\}
\end{aligned}
$$

In the above equations, $n 1$ and $n 2$ indicate, respectively, the numbers of channel the refrigerant flows into and out of the compression chamber, which include valve of the charge gas, valve of the discharge gas and the leakage path; $m_{g}$ and $m_{l}$ masses of the refrigerant and the oil respectively; $\dot{m}_{g i k}$ and $\dot{m}_{g o k}$ the inlet and outlet mass flow rate of the refrigerant; $\dot{m}_{l i}$ and $\dot{m}_{l o}$ the inlet and outlet mass flow rate of the oil; $T_{g}$ and $T_{l}$ temperature of the refrigerant and the oil; $i_{\text {gik }}$ and $C_{\mathrm{v}, g}$ indicate inlet entropy and specific heat of the refrigerant; $\rho_{l}$ the density of the oil; $V, h$ and $A$ volume, height and area of the control volume; $\theta_{m}$ and $\omega_{m}$ the rotating angle and rotating speed of the main shaft respectively. Based on the same argument, the governing equations for the lubricant are

$$
\frac{d m_{l}}{d \theta_{m}}=\frac{1}{\omega_{m}}\left(\sum_{k=1}^{n 3} \dot{m}_{l i k}-\sum_{k=1}^{n 4} \dot{m}_{l o k}\right)
$$

and

$$
\frac{d T_{l}}{d \theta_{m}}=\frac{1}{\omega_{m} m_{l} C_{v, l}}\left\{C_{v, l} \sum_{k=1}^{n 3}\left(T_{l i k}-T_{l}\right) \dot{m}_{l i k}-h A\left(T_{l}-T_{g}\right)\right\}
$$

in which $n 3$ and $n 4$ indicate, respectively, the numbers of channel the lubricant flows into and out of the compression chamber; $\dot{m}_{l i k}$ and $\dot{m}_{l o k}$ represent the inlet and outlet mass flow rate of the oil.

\section{LUBRICATION THEORY}

For an unequally matched scroll compressor with a centrifugal oil injection system as that shown in Fig. 1, oil is injected into the compression room with an inclined angle. Thus, the design of backpressure chamber for the fixed scroll is important. In general, the oil lubrication process of a scroll compressor begins with the lubrication of bearings by the oil from an oil tank. After that, some oil injects into the compressor room to seal and transfer heat with refrigerant, and the rest returns to the oil tank directly. For a complete lubrication theory, the following items should be accounted for.

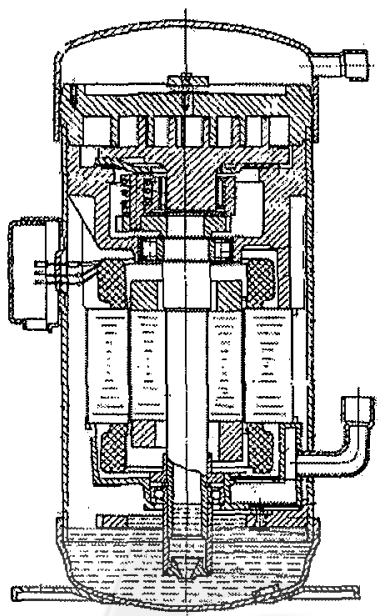

Fig. 1 Profile of a scroll compressor 


\subsection{Oil Loop}

In general, the oil flow rate of a centrifugal oil supply system is calculated as follows:

$$
Q=\frac{\pi r^{4}}{8 \rho \eta}\left(\frac{R \omega^{2}}{2}-\frac{g H}{R}\right)
$$

in which $Q$ is the flow rate of oil, $r$ the radius of oil orifice, $\rho$ the oil density, $\eta$ the dynamic viscosity of oil, $R$ the distance between the axial orifice and centerline of the main shaft, $\omega$ the rotating speed of the main shaft, $g$ the gravitational constant, and $H$ the distance of oil level in the oil tank counting from the oil inlet orifice. Without any ambiguity, many subscript notations are neglected here and hereafter for simplicity.

\subsection{Oil Temperature}

Oil temperature is one of the vital factors that affect the performance and dynamic load of a scroll compressor. Since slide bearing is usually used for a scroll compressor, the film flow lubrication model [5] is adopted herein to account for the oil temperature. It states that

$$
T=T_{i}+\frac{K_{1} K_{2} W_{v}}{Q C}
$$

in which $T_{i}$ indicates the temperature of the entrance oil, $K_{1}$ and $K_{2}$ the percentages of heat taken out and reserved in the thin film respectively, $W_{\nu}$ the dynamic loss of bearing lubrication, and $C$ the specific heat of oil. Combining Eqs. (5) and (6), one can find that oil temperature increases as the viscosity in Eq. (5) increases, as that shown by the dashed curve in Fig. 2 . However, according to the famous Rolelands formula [5] shown by the solid curve in Fig. 2, the oil viscosity should decrease as the temperature increases. In order to conform to both models to obtain the solution of the oil temperature, the intersection point of the two curves should be selected in the numerical calculation.

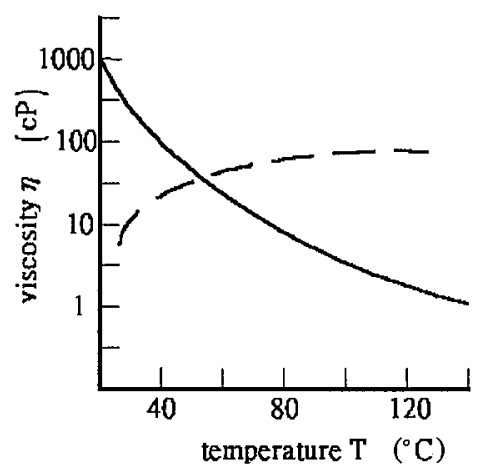

Fig. 2 Relationship between oil temperature and viscosity

\subsection{Heat Transfer Model}

The major function of the lubricant is to cool down the high temperature of the refrigerant. Therefore, heat transfer becomes one important factor that influences the performance of a scroll compress or. Unfortunately, the coefficient and area of heat transfer between the lubricant and the gas is not easy to decide. In the present study, the following two heat transfer mechanisms are considered. First, when the compression room encounters with the oil injection process, the individual lubricant drop is assumed to be spherical. Under this circumstance, the heat transfer coefficient between the lubricant and the gas is equivalent to that between the spheroid and the fluid flow. Alternately, when the compression room does not encounter with the oil injection process, since most lubricant adheres to the scroll body, the heat transfer between the lubricant and the gas behaves like a gas flow over a surface.

\subsection{Performance}

There are several definitions of efficiency used to evaluate the performance of a scroll compressor. The first one is the volumetric efficiency defined as the ratio between the real discharge flow rate and the theoretical discharge flow rate. The second one is the isothermal efficiency defined as the ratio between the isothermal compression work and the real compression work. The isothermal compression work can be calculated from

$$
W_{T}=D T_{s} \ln \frac{P_{d}}{P_{s}}
$$

in which $D$ indicates gas constant, $T_{s}$ and $P_{s}$ suction gas temperature and pressure, and $P_{d}$ is discharge gas pressure. The third frequently used efficiency is the compression efficiency defined as the ratio between the isothermal (or isentropic) compression work and the input shaft work. For the performance evaluation of an oil-injected compressor, the isothermal efficiency is usually used. Its difference from the compression efficiency lies in the fact that the latter includes more of the dynamic loss since the input shaft work is the summation of the real compression work and the dynamic loss.

\section{DYNAMIC LOAD ANALYSIS}

The dynamic load exerting on the orbiting scroll is affected by other load-acted components in a scroll compressor. These load-acted components include the fixed scroll, Oldham ring, eccentric shaft, and so on. In the present section, we shall establish relations among these load-acted components and utilize a numerical method to obtain these loads.

The dynamic load of the orbiting scroll comes mainly from the compressed gas force and the contact force. It can be divided into the axial, radial and tangential forces, respectively. The axial force is the projection of gas force in the axial direction. The radial force is 
the projection of gas force in the direction of a line connecting centers of the orbiting and fixed scrolls. And the tangential force is the projection of gas force in a direction normal to the above direction. Following the entire compression process, the gas force can be calculated in three different stages: before-mesh, during-mesh, and after-mesh. In the stages of before-mesh and after-mesh, it can be calculated from

$$
F_{s}(\theta)=\sum_{k=1}^{N+1} P_{k} S_{k}(\theta)
$$

for the axial force,

$$
F_{l}(\theta)=\sum_{k=1}^{N} 2 \pi b h\left(2 k-\frac{\theta}{\pi}\right)\left(P_{k}-P_{k+1}\right)
$$

for the tangential force, and

$$
F_{r}(\theta)=2 b h\left(P_{1}-P_{N+1}\right)
$$

for the radial force. In the above equations, $\theta$ is rotating angle of the main shaft, $S$ the projection area, $N$ the number of pressure chamber, $b$ radius of the base circle, and $h$ thickness of the scroll body.

During the stage of mesh, the axial force can be calculated from Eq. (8) by using Green's theorem [6], and the tangential and radial forces can be obtained from

$$
F_{t}(\theta)=L_{t}(\theta) h\left(P_{1}-P_{2}\right)+\sum_{k=2}^{N} 2 \pi b h\left(2 k-\frac{\theta}{\pi}\right)\left(P_{k}-P_{k+1}\right)
$$

and

$$
F_{r}(\theta)=L_{r} h\left(P_{1}-P_{2}\right)+2 b h\left(P_{2}-P_{N+1}\right)
$$

respectively. In the above equations, $L_{t}$ indicates the line of mesh projected in a direction connecting centers of the two base circles and $L_{r}$ represents the same line of mesh but projected in the plane perpendicular to the above direction. At a certain point of interest; the resultant forces can be calculated from the combination of radial and tangential forces. For the eccentric shaft of the orbiting scroll, since the force does not locate at the same plane as the applied point of the resultant force, an overturning moment has the tendency to overturn the orbiting scroll. The overturning moment is calculated as

$$
M_{t}=\sqrt{F_{t}^{2}+F_{r}^{2}}\left(\frac{h}{2}+h_{1}\right)
$$

in which $h_{1}$ is the distance between the eccentric shaft-driven plane and the plane exerted on by the gas force. In addition, there will be a spinning moment exerted on the eccentric shaft by the orbiting scroll. It arises from the tangential gas force exerting on the middle point of a line-segment between the orbiting and fixed scrolls. This spinning moment can be calculated as

$$
M_{r}=\frac{r}{2} P_{s} p h \sum_{k=1}^{N}\left(2 k-\frac{\theta}{\pi}\right)\left(\rho_{k}-\rho_{k+1}\right)
$$

in which $P_{s}$ the suction pressure, $p$ pitch of the scroll body, $\rho_{k}$ the pressure ratio, and $r$ the distance between centers of the two base circles.

The major contribution of the contact force to the orbiting scroll is the centrifugal force. It arises from rotation of the orbiting scroll. Since the mass center does not coincide with the geometric center of the orbiting scroll, a resultant centrifugal force can be calculated as

$$
F_{\mathrm{Im}}=m_{\mathrm{Im}} L_{\mathrm{Im}} \omega^{2}
$$

in which $m_{\mathrm{Im}}$ indicates mass of the orbiting scroll and $L_{\mathrm{Im}}$ represents the eccentric distance of the orbiting scroll.

The main shaft is subjected to both bending and twisting moments. The bending moment arises from the fact that resultant force of the orbiting scroll does not lie on the same plane as the driving force of the generator. It can be calculated from

$$
M_{B}=L_{b} \sqrt{F_{t}+\left(F_{\mathrm{Im}}-F_{r}\right)}
$$

in which $L_{b}$ is the distance between the bearing and the acting point of force. Moreover, since the centerline of the main shaft does not lie on the same plane as that of the eccentric shaft, a twisting moment also arises. It can be calculated from

$$
M_{e}=\frac{W_{z}}{\omega}
$$

where $W_{z}$ indicates the input work of the compressor. Hence, the resultant moment exerting on the main shaft is

$$
M=\sqrt{M_{B}^{2}+M_{e}^{2}}
$$

The force exerting on the Oldham ring should be examined carefully as well. Forces resulting from the casing and the orbiting scroll are major loads for the Oldham ring. A thermal-fluid model [7] is adopted in the present study to simulate the gas compression process of the Oldham ring. Equations used in the calculation are referred to $\mathrm{Li}[8]$.

\section{MODEL TESTING AND PERFORMANCE SIMULATION}

Based on the above theoretical analysis, a computer program founded primarily on the fourth-order Runge-Kutta method is written to simulate the performance of a scroll compressor. Experimental work has also been carried out to examine the accuracy of the analytical model. The experimental data are documented in detail in Hsieh and Chen [9]. In the present paper, some comparisons are made between the analytical results and the experimentally measured data. 
In particular, the result of the refrigerant flow rate, discharge temperature and input work are compared and shown in Table 1. As indicated by the small discrepancies between the theoretical and experimental results for all categories, the accuracy of the present model can somewhat be justified.

Table 1 Comparison between calculated and experimental results

\begin{tabular}{|c|c|c|c|c|c|c|}
\hline $\begin{array}{c}\text { Suction } \\
\text { Temp. } \\
\left({ }^{\circ} \mathrm{C}\right)\end{array}$ & $\begin{array}{c}\text { Suction } \\
\text { Pressure } \\
(\mathrm{bar})\end{array}$ & $\begin{array}{c}\text { Discharge } \\
\text { Pressure } \\
(\mathrm{bar})\end{array}$ & $\begin{array}{c}\text { Refrigerant } \\
\text { flow rate } \\
(\mathrm{kg} / \mathrm{min})\end{array}$ & $\begin{array}{c}\text { Discharge } \\
\text { Temp. } \\
\left({ }^{\circ} \mathrm{C}\right)\end{array}$ & $\begin{array}{c}\text { Input } \\
\text { Work } \\
(\mathrm{kW})\end{array}$ \\
\hline 18 & 5.33 & 20.8 & Exp. & 16.4 & 99.4 & 14.214 \\
\hline & & & Sim. & 16.35 & 101 & 15.75 \\
\hline & & $\begin{array}{c}\text { Difference } \\
(\%)\end{array}$ & 0.36 & 1.579 & 9.75 \\
\hline 10 & 4.03 & 18.7 & Exp. & 13.3 & 99.8 & 12.786 \\
\hline & & & Sim. & 13.1 & 92.5 & 12.95 \\
\hline 9.9 & 4.05 & 14.5 & Exp. & 13.2 & 83.0 & 10.85 \\
\hline & & & Sim. & 13.18 & 77.9 & 10.748 \\
\hline & & & $\begin{array}{c}\text { Difference } \\
(\%)\end{array}$ & 0.15 & 6.12 & 0.94 \\
\hline
\end{tabular}

After the theoretical model has been justified, more detailed performance and dynamic load analysis are carried out. Some of the results are shown in Figs. 3 to 12. Among them, Fig. 3 indicates the variation of temperature, pressure and control volume in the compression room with respect to the rotating angle of the main shaft. It can be seen from the figure that the control volume becomes larger and larger as the position of the control system, not wrapped by the fixed and orbiting scrolls, moves toward the discharge gas hole. After reaching its peak, the control volume becomes smaller and smaller as the position of the control system, wrapped by the fixed and orbiting scrolls, proceeds to the discharge gas hole. Before the position of the control system is wrapped by the fixed and orbiting scrolls, the increase of temperature and pressure is not so obvious. Significant increase of temperature and pressure occurs only after the control volume has reached its peak and moves gradually to the discharge hole.

To study the relationship between compression ratio and build-in volume ratio on the compressor performance, Fig. 4 is constructed. It can be seen that, for a fixed build-in volume ratio, the volumetric efficiency decreases as the compression ratio increases. It is attributed to the leakage caused by the gradually increased pressure. Unlike the volumetric efficiency, the compression efficiency exists an optimum value at a certain compression ratio. This compression ratio is usually equal to the design compression ratio. It can also be seen that, for a fixed compression ratio, there exists optimum compression efficiency at a certain build-in volume ratio.

Figure 5 investigates the influence of oil level on oil flow rate for various oil-hole diameters and rotating speeds. As shown in this figure, oil flow rate increases as the rotating speed increases or the oil-hole diameter is enlarged. The distance between oil level and oil

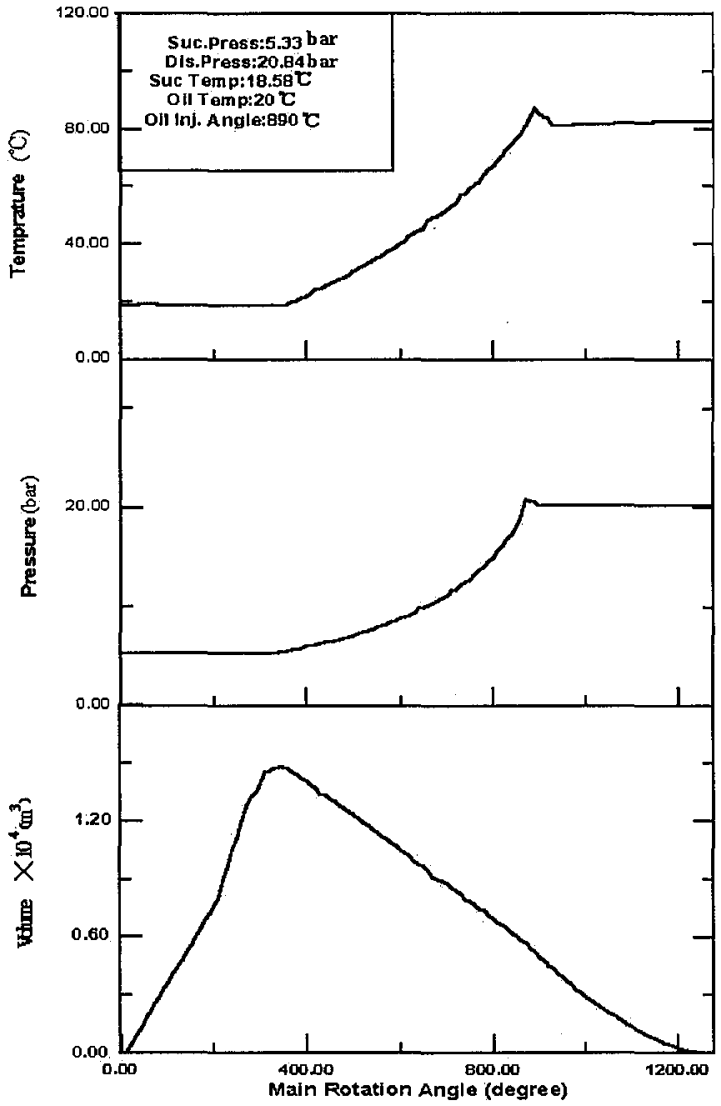

Fig. 3 Physical properties of compression room during compression process
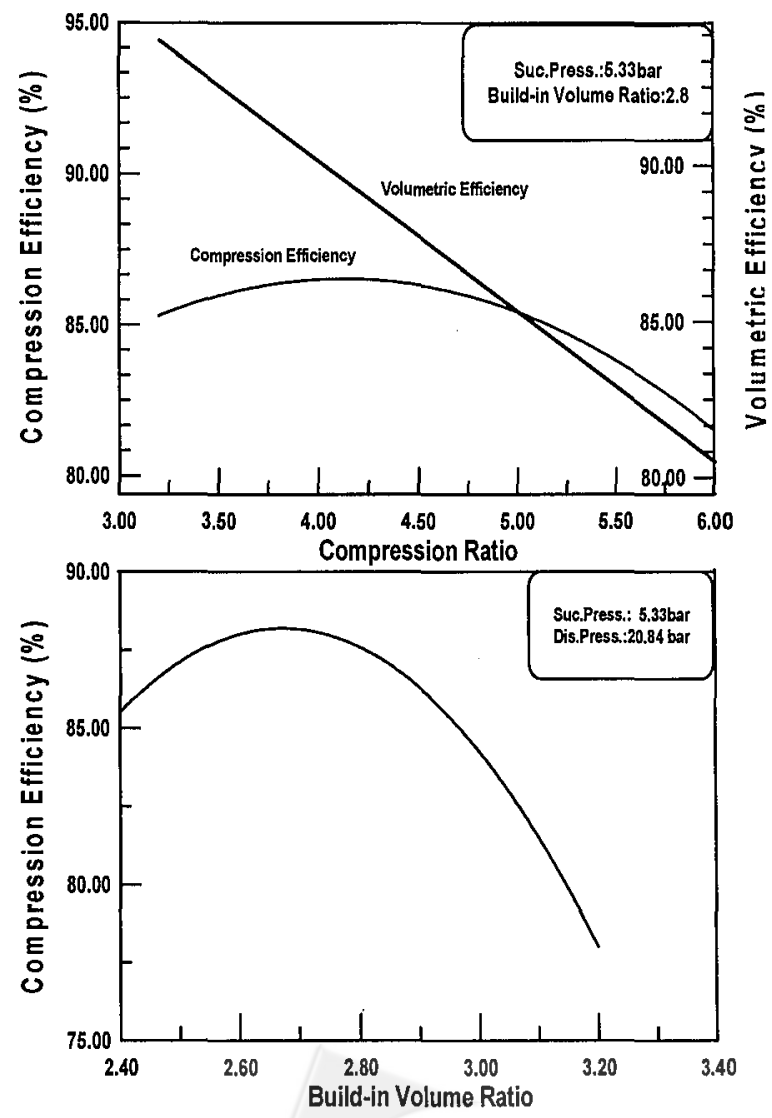

Fig. 4 Relationship between oil compression ratio and build-in volume ratio 
inlet orifice also increases as the oil flow rate decreases. The larger centrifugal force caused by the higher rotating speed and the lower gravity owing to lower oil level are two possible reasons for the resultant of lower oil flow rate. For oil holes, it is very obvious that a larger diameter usually lets more oil pass through and results in a higher oil flow rate. With regard to the dynamic loads, Figs. 6 and 7 indicate that there is fluctuation of gas force and moment appearing gradually in the mesh region between $70^{\circ}$ to $170^{\circ}$. It is because that several compression chambers have already encountered with the discharge gas hole. In fact, the same fluctuation trend is found for the contact force as it can be seen from Fig. 8. It appears to be a sine wave but influenced by loads coming from the Oldham ring. For parametric study, Figs. 6 to 8 indicate the influence of rotating speed on the gas force; gas moment (including that on the main shaft) and contact force. The parameters Fn3 and Fn4 in Fig. 8 indicate the contact forces exerting on the Oldham ring, and $\mathrm{Fz}$ is the contact force exerting on the orbital scroll. These forces were obtained through solving numerically a series of equilibrium equations [7]. In most of the above cases, dynamic loads increase as the rotating speed increases in the fluctuation region. It indicates

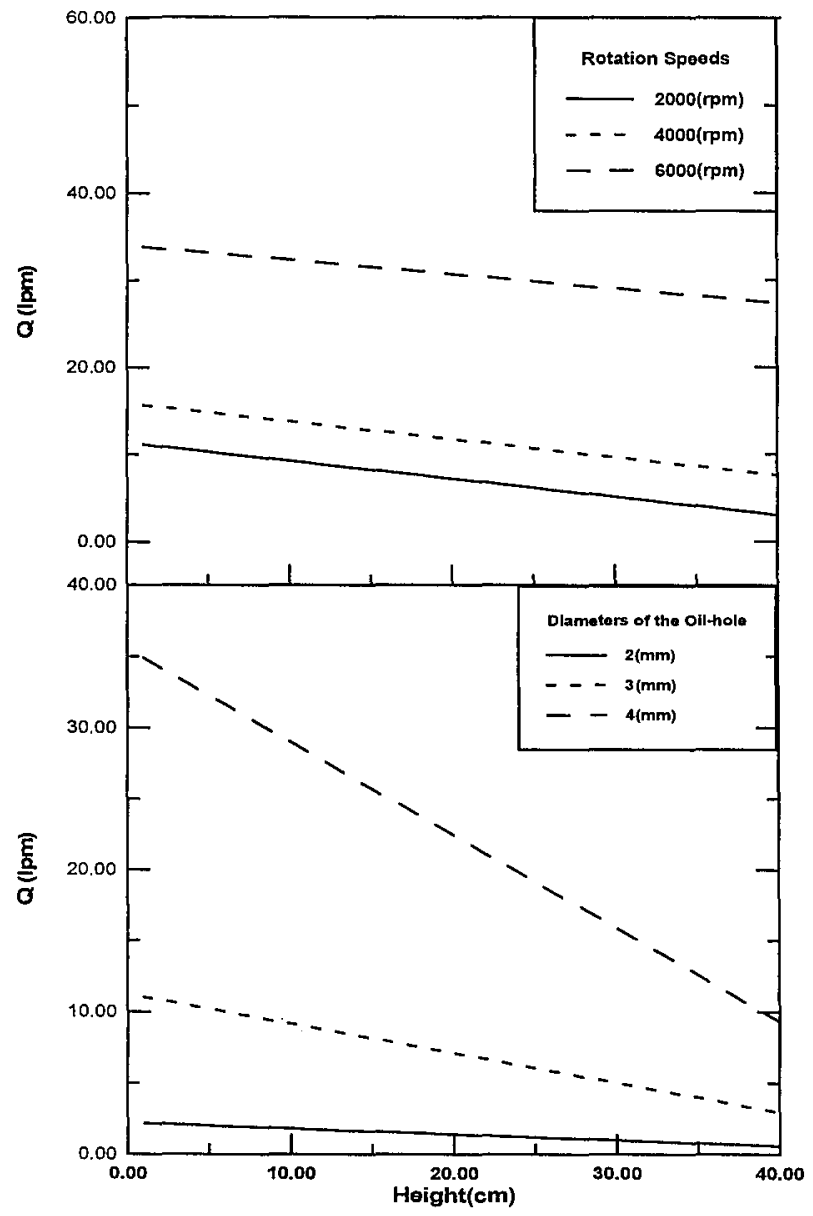

Fig. 5 Effect of oil level on oil flow rate that a higher rotating speed does not only increase the oil flow rate but also decrease the chance of leakage and, therefore, results in larger values of forces and moments. In fact, these dynamic loads are also influenced by other parameters such as oil flow rate, oil inlet temperature and oil injection position. However, their influences are not so obvious as that of the rotating speed. Therefore, the results are not presented herein. To let the readers have a closer look, a portion of curves is enlarged and enclosed by a quadrangle at the left hand side of Figs. 6 to 8 and figures hereafter.

Figure 9 shows the effect of oil flow rate on the performance and dynamic loads of a scroll compressor. Both compression and volumetric efficiencies increase but the gas force decreases as the oil flow rate increases. This is attributed to the lower mean pressure due to descending temperature in the compression process. The lower mean pressure causes less leakage and leads to higher volumetric efficiency. It also causes less energy loss and generates smaller wrapped area in the pressure-volume diagram of the refrigerant during the compression process, and leads to the higher compression efficiency. The analysis of contact force and moment appears to have a similar result as before and is neglected herein for the sake of simplicity.

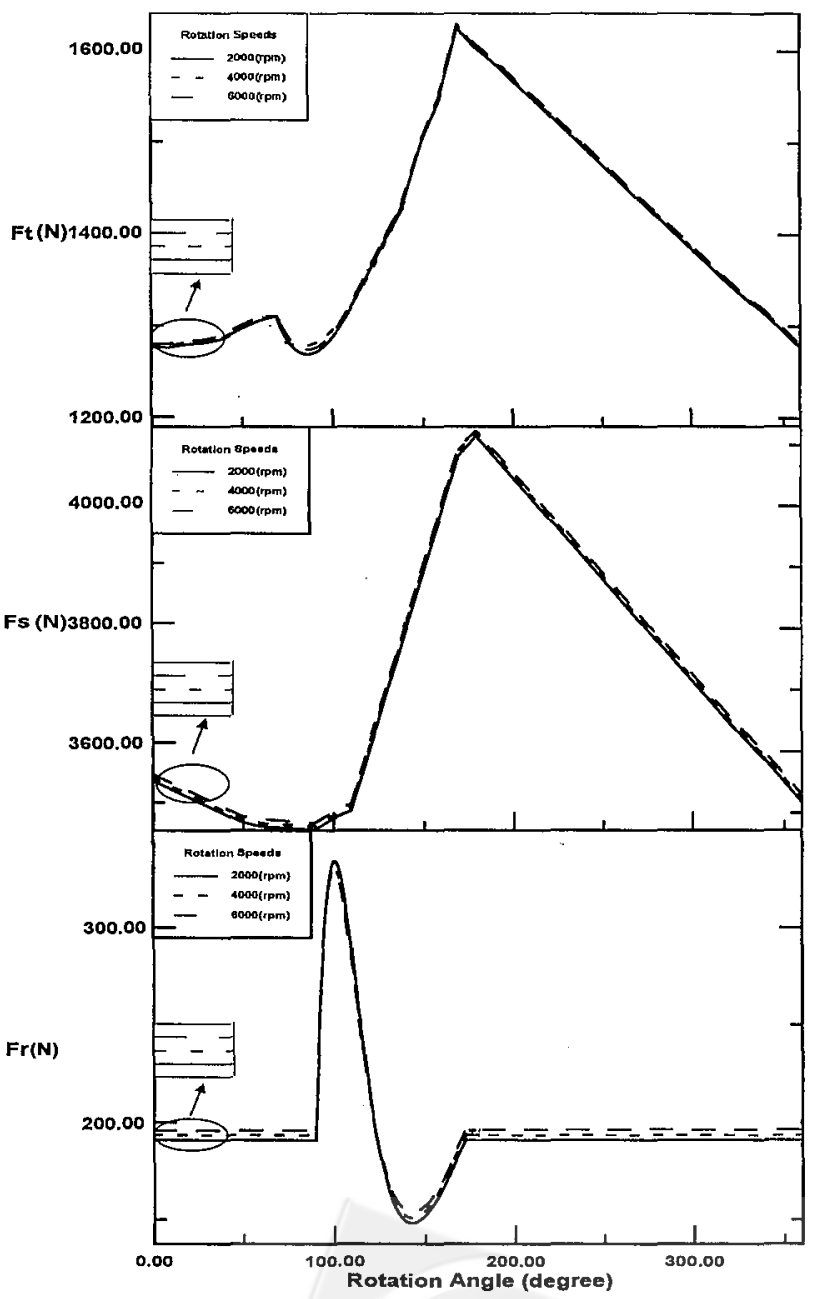

Fig. 6 Fluctuation of gas force 


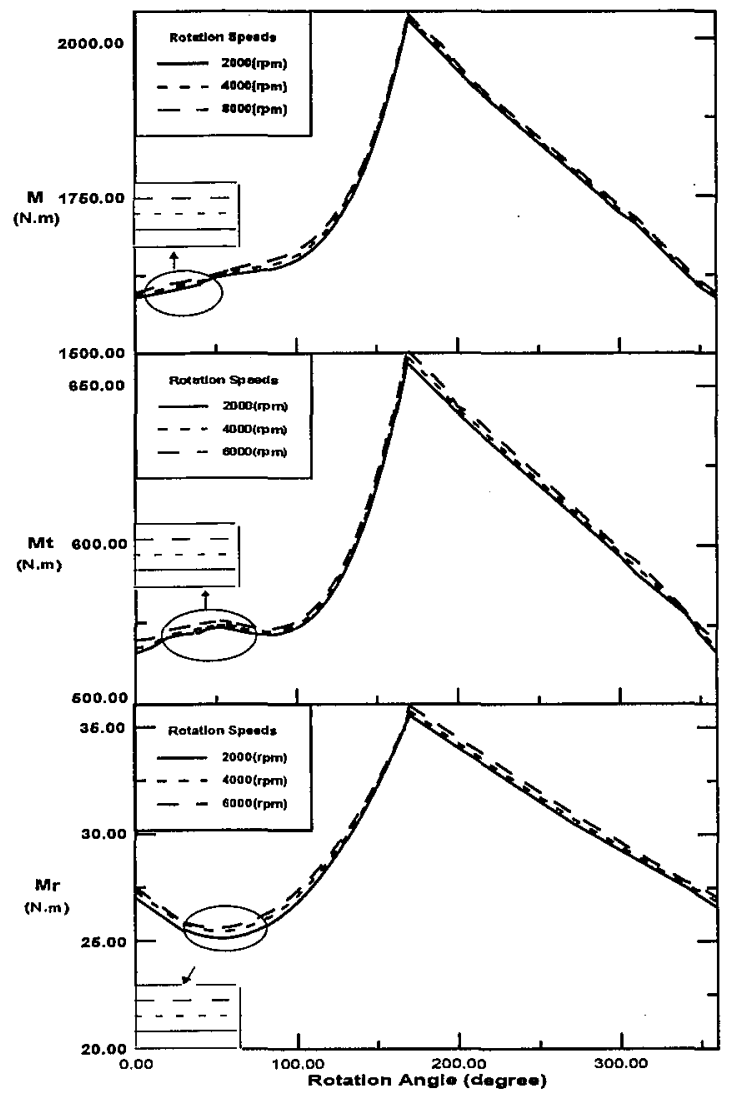

Fig. 7 Fluctuation of gas moment
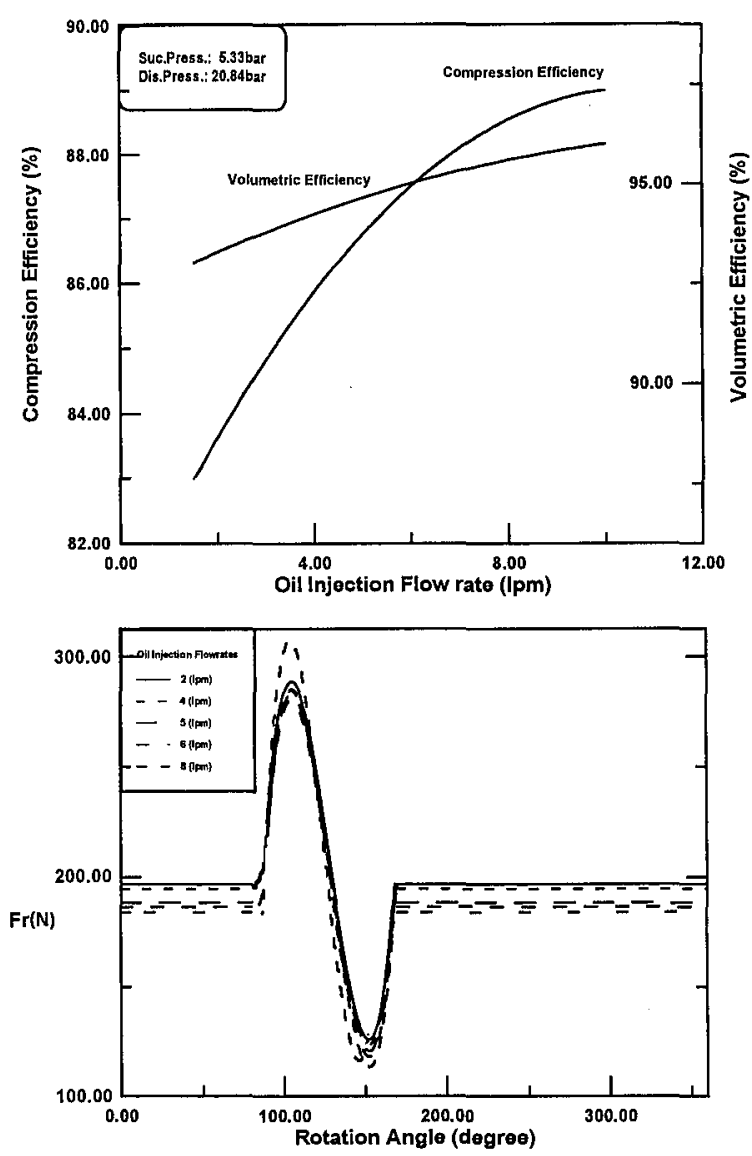

Fig. 9 Effect of oil flow rate

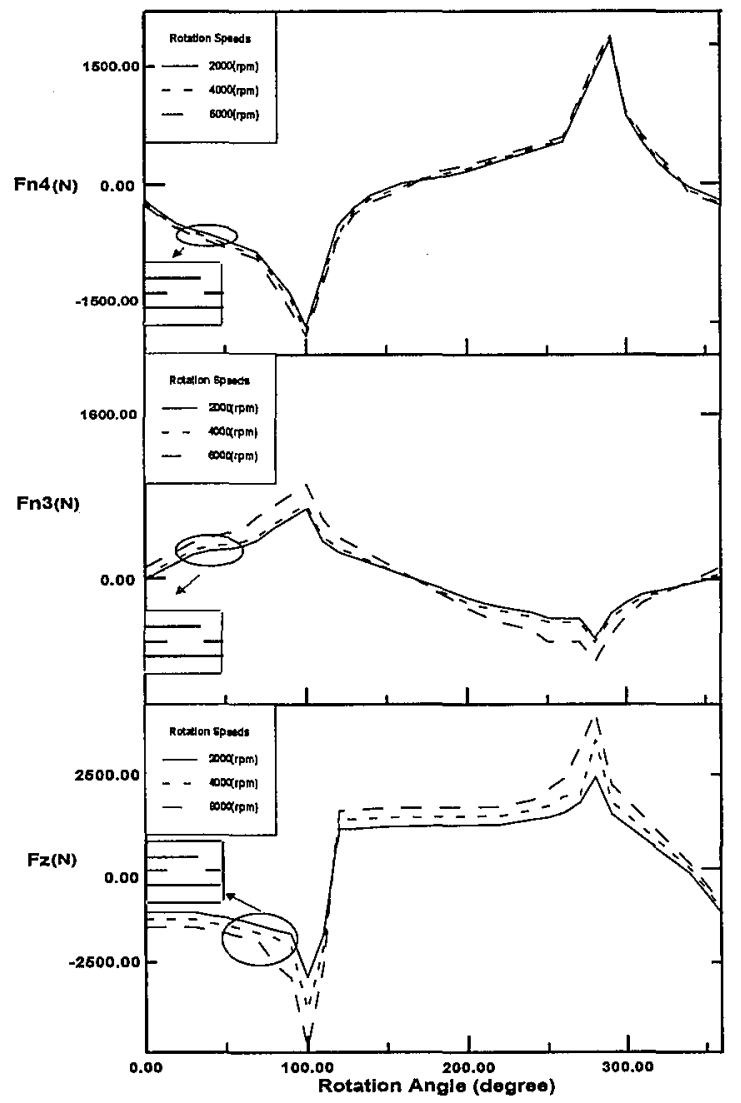

Fig. 8 Fluctuation of contact force

The influence of oil inlet temperature on the performance and dynamic loads is demonstrated in Fig. 10. The volumetric efficiency decreases but the gas force increases as the oil inlet temperature increases. The increase of gas force is owing to that, at a higher oil inlet temperature, both gas pressure and gas force are more difficult to be lowered down. As for the decrease of volume efficiency, it is because that oil viscosity decreases as the temperature increases. However, when heat transfer effect is taken into consideration, the situation may be different. The result is that there exists an optimum oil injection temperature associated with the maximum compression efficiency as that shown in Fig. 10. As for the analysis of contact forces and moments, a similar procedure is followed and similar results are obtained as before.

Figure 11 indicates the influence of oil injection position on the performance and dynamic loads of a scroll compressor. Gas force increases as the oil injection position is moved closer to the discharge side. This is because that temperature effect occurs too late and, therefore, the gas pressure in the compression room cannot be reduced effectively in time. Both the volumetric and compression efficiencies decrease as the oil injection position is moved closer to the discharge side. Again, it is because that the oil seal and heat transfer effects occur too late, and the distribution of the pressure remains too high in the compression room. 

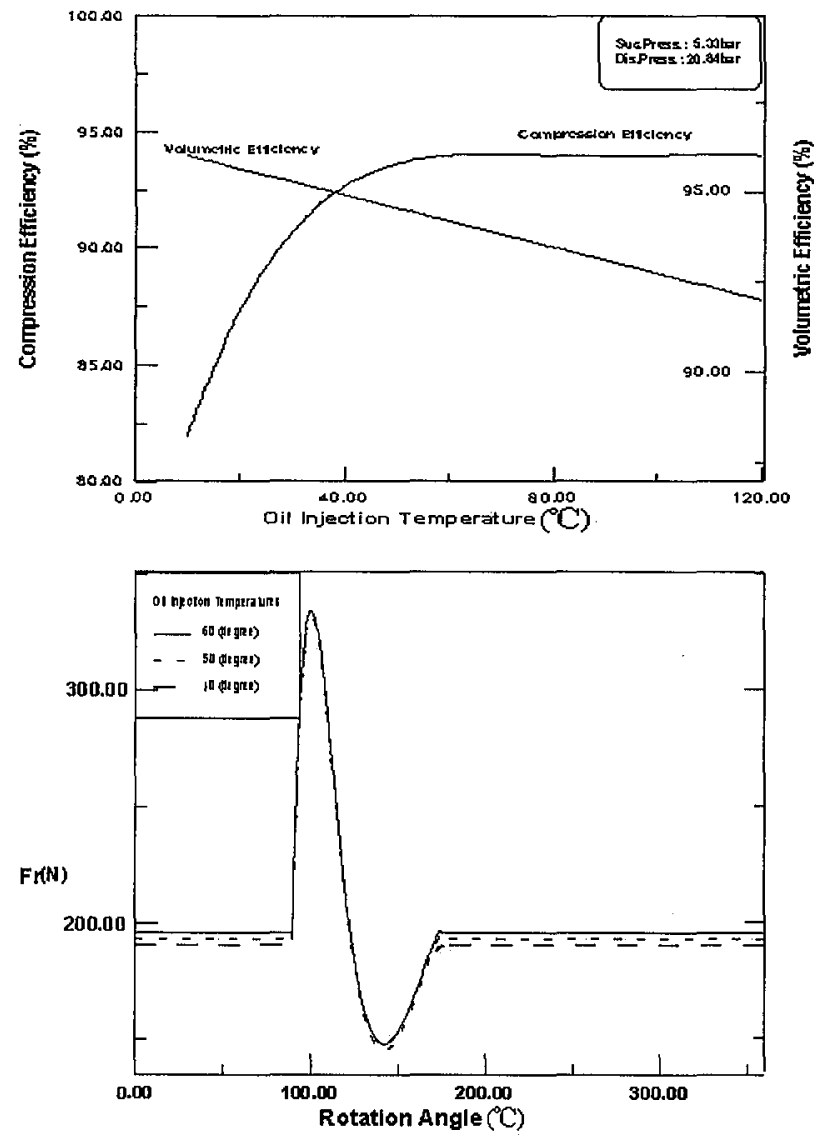

Fig. 10 Effect of oil inlet temperature
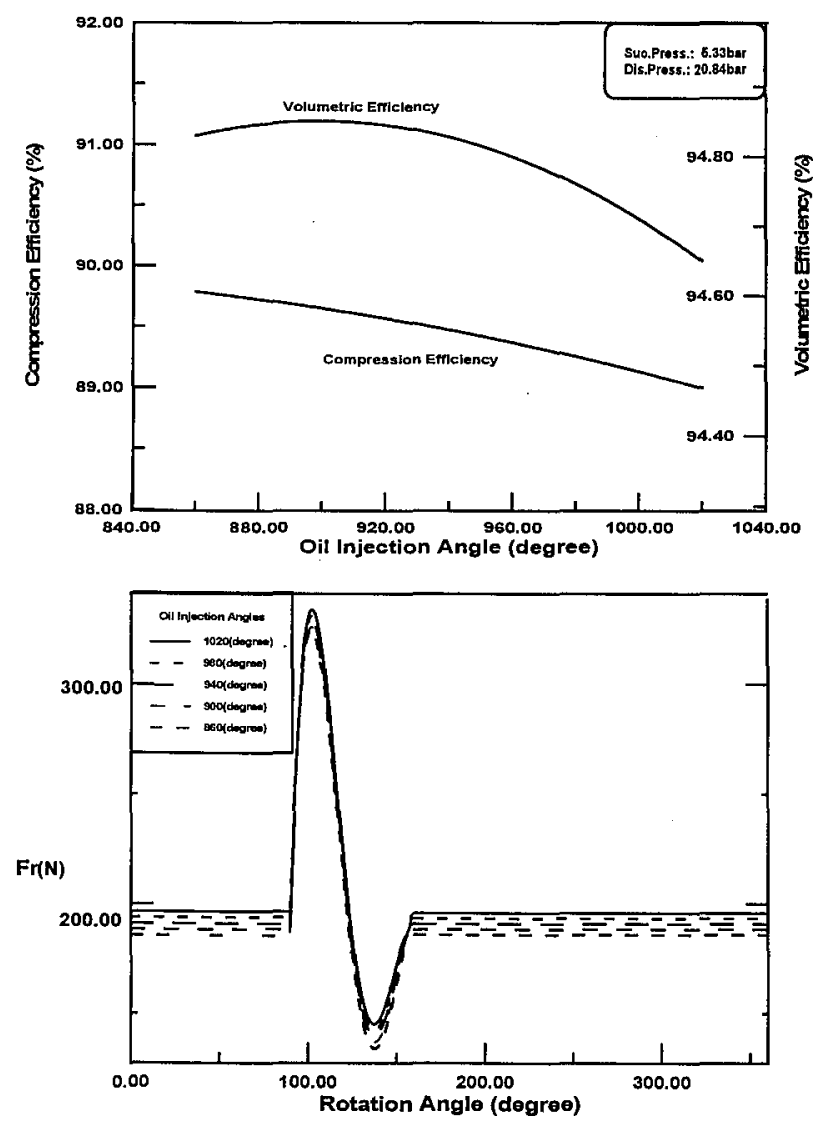

Fig. 11 Effect of oil injection position
Figure 12 studies the effect of rotating speed on the performance and dynamic loads of a scroll compressor. It is found that both the volumetric efficiency and dynamic loads increase as the rotating speed increases. The reasons have already been mentioned before. On the other hand, the compression efficiency decreases as the rotating speed increases. The reason is that fast rotating speed usually generates high discharged pressure and results in much energy loss.
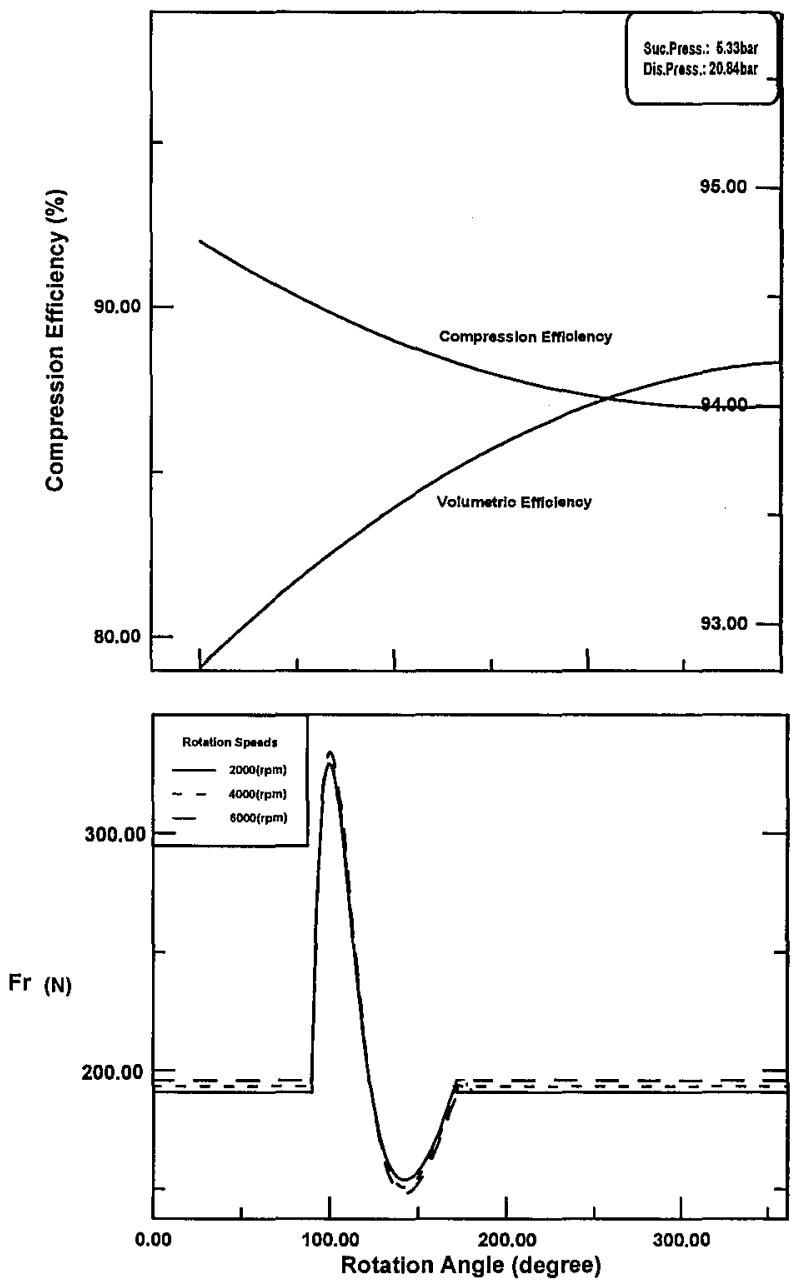

Fig. 12 Effect of rotating speed

\section{CONCLUSION}

A theoretical model for the prediction of thermal performance and dynamic loads of a scroll compressor is developed in this paper. The preliminary theoretical prediction agrees very well with the experimental data, and justifies the accuracy of the analytical model. More detailed calculations are then performed and some of the results have been presented. The following summarized conclusions can be drawn at the end of the present paper:

(1) The fixed scroll wrap of a scroll compressor decides the special build-in volume ratio and the compression ratio of the system. In general, the 
best compression efficiency occurs at a compression ratio comparable to the design compression ratio.

(2) Both the volumetric and compression efficiencies increase as the flow rate increases. However, the dynamic loads decrease as the oil flow rate increases.

(3) The volumetric efficiency decreases as the oil inlet temperature increases. As for the compression efficiency, there exists an optimum oil injected temperature that results in the maximum efficiency.

(4) The dynamic loads increase as the oil inlet temperature increases. Both the performance and dynamic loads increase as the oil injection position is moved closer to the discharge side.

(5) Both the volumetric efficiency and dynamic loads increase as the rotating speed increases, but the compression efficiency has the opposite trend. The rotating speed is the most influential parameter in the design of a scroll compressor.

(6) The fluctuation of contact force is more vibrant than that of the gas force.

\section{REFERENCES}

1. Gu, Y. and Wu, Y., "Experimental Investigation on Heat Transfer in the Manifold of Refrigerating Compressors," Proceedings of the International Compressor Engineering Conference, Purdue, West Lafayette, pp. 175-186 (1986).

2. Meyer, W. A. and Thompson, H., "An Experimental Investigation into Heat Transfer to the Suction Gas in a Low-Side Hermetic Refrigeration Compressor," Proceedings of the International Compressor Engineering Conference, Purdue, West Lafayette, pp. 477-488 (1988).

3. Suefuji, K., Shiibayashi, M. and Tojo, K., "Performance Analysis of Hermetic Scroll Compressors," Proceedings of the International Compressor Engineering Conference, Purdue, West Lafayette, pp. 75-84 (1992).

4. Short, G. D. and Rajewski, T. E., "Refrigeration Lubricants-Current Practice and Feature Development," Proceedings of the International Compressor Engineering Conference, Purdue, West Lafayette (1996).

5. Sun, D. C., "Equation Used in Hydro Dynamic Lubrication," Journal of the Society of the Tribologists and Lubrication Engineers, pp. 18-25 (1997).

6. Lee, Y. R., "Geometric, Thermal-Fluid and Dynamic Analysis of Screw and Scroll Compressors," Ph.D. Dissertation, Department of Mechanical Engineering, National Taiwan University, Taipei, Taiwan (1996).

7. Lin, S. S., "Performance Simulation and Gas Force Analysis of Scroll Compressors," Master Thesis, Department of Mechanical Engineering, National Taiwan University, Taipei, Taiwan (1999).

8. Li, L. S., "Scroll Compressor," Mechanical Industry, China (1998).

9. Hsieh, J. J. and Chen, S. L., "Dynamic, Lubrication and Heat Transfer Analysis of Scroll Compressors," Technical Report, Department of Mechanical Engineering, National Taiwan University, Taipei, Taiwan (2000).

\section{NOMENCLATURE}

$A:$ area $\left[\mathrm{m}^{2}\right]$

$b \quad:$ radius of base circle $[\mathrm{m}]$

$C:$ specific heat $[\mathrm{kJ} / \mathrm{kg} \cdot \mathrm{K}]$

$\mathrm{D}$ : gas constant $[\mathrm{kJ} / \mathrm{kg} \cdot \mathrm{K}]$

$F:$ force $[\mathrm{N}]$

$g$ : gravitational constant $\left[\mathrm{m} / \mathrm{s}^{2}\right]$

$h:$ height or thickness [m]

$H$ : distance of oil level in oil tank counting from oil inlet orifice [m]

$i:$ inlet entropy $[\mathrm{kJ} / \mathrm{kg} \cdot \mathrm{K}]$

$K_{1}$ : heat ratio taken out from the film

$K_{2}:$ heat ratio kept in the film

$L \quad$ : length [m]

$m:$ mass $[\mathrm{kg}]$

$M:$ moment $[\mathrm{N} \cdot \mathrm{m}]$

$n 1$ : number of channel flow into compression room for gas

$n 2:$ number of channel flow out of compression room for gas

$n 3$ : number of channel flow into compression room for oil

$n 4:$ number of channel flow out of compression room for oil

$P \quad:$ pressure or pitch of scroll body [bar]

$Q$ : oil flow rate $[\mathrm{kg} / \mathrm{s}]$

$r$ : radius of oil orifice [m]

$R$ : distance between axial orifice and centerline of main shaft [m]

$S:$ projection area $\left[\mathrm{m}^{2}\right]$

$T$ : temperature $[\mathrm{K}]$

$u$ : flow velocity $[\mathrm{m} / \mathrm{s}]$

$V:$ volume $\left[\mathrm{m}^{3}\right]$

$W:$ work $[\mathrm{J}]$

\section{Greek symbols}

$\delta:$ thickness of clearance [m]

$\phi:$ : inclined angle of oil pipe at oil orifice $\left[{ }^{\circ} \mathrm{C}\right]$

$\eta:$ dynamic viscosity of oil $\left[\mathrm{N} \cdot \mathrm{s} / \mathrm{m}^{2}\right]$

$\theta$ : rotating angle of main shaft $\left[{ }^{\circ} \mathrm{C}\right]$

$\rho:$ density $\left[\mathrm{kg} / \mathrm{m}^{3}\right]$

$\omega:$ rotating speed of main shaft $[\mathrm{rad} / \mathrm{s}]$

\section{Subscripts}

$a$ : sealing

$b \quad:$ application plane and/or driving plane of gas force

$B$ : bending

$d$ : discharge

$e:$ rotation

$f$ : friction

$g$ : gas

$g i:$ inlet gas of control volume

go : outlet gas of control volume 
$i \quad:$ initial value or flow in

Im : mass of scroll body

$l:$ oil

li : inlet oil of control volume

lo : outlet oil of control volume

$m$ : main shaft

$m n:$ mean value

$N$ : normal direction

$o:$ oil pipe or flow out

$r$ : radial direction

$s:$ axial direction/or suction

$t:$ tangential direction

$v$ : bearing loss/or isovolume

$z$ : input of compressor

(Manuscript accepted for publication Nov. 23, 2001.) 